\title{
Continuidad en estructuras débiles generalizadas
}

\author{
Continuity in Generalized Weak Structures
}

J. Ávila ${ }^{\mathrm{a}, *}$

F. Molina ${ }^{b}$

Recepción: 30-abr-15

Aceptación: 07-jun-15

\begin{abstract}
Resumen
En este trabajo se hace un estudio de la continuidad en Estructuras Débiles Generalizadas (EDG), introduciendo la versión local y global de la misma. Además de mostrar varias caracterizaciones, extendemos varios resultados clásicos de topología, topologías generalizadas y estructruas minimales relacionados con axiomas de separación.
\end{abstract}

Palabras clave: topología generalizada, estructura minimal, estructura débil, estructura débil generalizada, función continua.

\begin{abstract}
In this paper we study the continuity in generalized weak structures and we extend to this setting the global and local continuity. We present several characterizations of continuity and we extend some classical topological results related to separation axioms.
\end{abstract}

Key words: Generalized topology, Minimal structure, Weak structure, Generalized wead structure, Continous function.

\footnotetext{
${ }^{a}$ Universidad del Tolima.

*Autor de correspondencia: javila@ut.edu.co

${ }^{\mathrm{b}}$ Universidad de Ibagué.
} 


\section{Introducción}

Desde el punto de vista matemático y teórico el estudio de nociones más generales que la estructura topológica ha permitido un desarrollo inusitado de la topología general, en los últimos 15 años. Las topologías generalizadas introducidas por Császár en 1997 [1], han mostrado ser una excelente generalización de topología, evidenciado en las innumerables publicaciones recientes relacionadas con el tema. Por otro lado las estructuras minimales ( $m$-estructruras o $m$-espacios) introducidas por Maki en 1996 [2] también constituyen otra, no menos importante línea de investigación en este campo, con importantes desarrollos también. Más recientemente Császár en 2011 [3] introdujo las estructuras débiles, las cuales buscan un contexto aún más general donde las nociones topológicas estudiadas en trabajos anteriores puedan seguir siendo válidas. En este sentido Ávila y Molina en 2012 [4] introdujeron las estructuras débiles generalizadas, considerando así el contexto más general posible donde pueden estudiarse las nociones topológicas conocidas. Es importante mencionar que nociones topológicas en colecciones fueron primeramente consideradas en 1999 [5], donde se hace un estudio general, pero amplio, de continuidad, compacidad, conexidad, estructuras iniciales y finales y de los axiomas de separación $T_{0}, T_{1}$ y $T_{2}$, entre otras. Aunque en ese trabajo la colección de los conjuntos considerados abiertos es cerrada para uniones, las definiciones de compacidad, separación, conexidad y continuidad se hacen teniendo en cuenta el mayor abierto contenido en el espacio lo cual conduce a resultados bastante interesantes y distintos de los usuales, pero que también generalizan aquellos resultados clásicos.

En este trabajo se generalizan algunos resultados sobre continuidad en $m$-espacios [6] y topologías generalizadas $[7,8,9]$. Introducimos las versiones local y global y observamos que no son equivalentes. Se presentan varias caracterizaciones de la versión local en términos del $\mathfrak{g}$-interior y la $\mathfrak{g}$-clausura y se extienden varios resultados que relacionan continuidad con espacios $\mathfrak{g}-T_{0}, \mathfrak{g}-T_{1}$ y $\mathfrak{g}-T_{2}$.

\section{Nociones básicas}

En esta primera parte se presentan algunos resultados y definiciones básicas que se necesitarán en las secciones posteriores. Estos conceptos y otros adicionales pueden consultarse en [4].

Definición 1. Una estructura débil generalizada (EDG) sobre un conjunto $X \neq \emptyset$, es una clase no vacía g de subconjuntos de $X$.

Si $\mathfrak{g}$ es una estructura débil generalizada sobre $X$, entonces al par $(X, \mathfrak{g})$ se le llama $\mathfrak{g}$-espacio o en algunos casos para simplificar lo llamaremos espacio. Cada elemento de $\mathfrak{g}$ se llama $\mathfrak{g}$-abierto y su complemento $\mathfrak{g}$-cerrado.

Al igual que en todos los otros casos citados de topologías, topologías generalizadas y las demás, es posible definir adecuadamente el interior y la clausura de cualquier subconjunto del espacio.

Definición 2. Sean $X$ un $\mathfrak{g}$-espacio y $A \subseteq X$. El $\mathfrak{g}$-interior y la $\mathfrak{g}$-clausura de $A$ se definen por $i_{\mathfrak{g}}(A)=$ $\cup\{U: U \subseteq A, U \in \mathfrak{g}\}$ y $c_{\mathfrak{g}}(A)=\cap\left\{F: A \subseteq F, F^{c} \in \mathfrak{g}\right\}$ respectivamente.

Nótese que en casos particulares podría tenerse que $i_{\mathfrak{g}}(X) \neq X$ y que $c_{\mathfrak{g}}(\emptyset) \neq \emptyset$. Las principales propiedades de estos operadores, en particular la idempotencia y la monotonía, se presentan a continuación [4].

Proposición 1. Sean g una $E D G$ sobre el conjunto $X$ y $A, B \subseteq X$. Entonces:

1. $i_{\mathfrak{g}}(A) \subseteq A$.

2. Si $A \in \mathfrak{g}$, entonces $i_{\mathfrak{g}}(A)=A$.

3. Si $A \subseteq B$, entonces $i_{\mathfrak{g}}(A) \subseteq i_{\mathfrak{g}}(B)$.

4. $i_{\mathfrak{g}}\left(i_{\mathfrak{g}}(A)\right)=i_{\mathfrak{g}}(A)$.

5. $A \subseteq c_{\mathfrak{g}}(A)$.

6. Si $A^{c} \in \mathfrak{g}$, entonces $c_{\mathfrak{g}}(A)=A$.

7. Si $A \subseteq B$, entonces $c_{\mathfrak{g}}(A) \subseteq c_{\mathfrak{g}}(B)$.

8. $c_{\mathfrak{g}}\left(c_{\mathfrak{g}}(A)\right)=c_{\mathfrak{g}}(A)$.

Proposición 2. Sean g una $E D G$ sobre el conjunto $X$ y $A \subseteq X$. Entonces:

1. $i_{\mathfrak{g}}(A)^{c}=c_{\mathfrak{g}}\left(A^{c}\right)$.

2. $i_{\mathfrak{g}}\left(A^{c}\right)=c_{\mathfrak{g}}(A)^{c}$.

3. $x \in i_{\mathfrak{g}}(A)$, si y sólo si, existe $U \in \mathfrak{g}$ tal que $x \in U$ y $U \subseteq A$.

4. $x \in c_{\mathrm{g}}(A)$, si y sólo si, para todo $U \in \mathfrak{g}$ tal que $x \in U$ se tiene $U \cap A \neq \emptyset$. 


\section{Continuidad en EDG'S}

Ahora generalizamos las nociones de función ( $\left.m, m^{\prime}\right)$-continua y de continuidad generalizada dadas en [6] y [7] respectivamente.

Definición 3. Sean $\mathfrak{g}$ y $\mathfrak{g}^{\prime} E D G$ 's sobre $X$ e $Y$ respectivamente. Se dice que una función $f: X \rightarrow Y$ es $\left(\mathfrak{g}, \mathfrak{g}^{\prime}\right)$-continua, si para cada $V \in \mathfrak{g}^{\prime}$ se tiene que $f^{-1}(V) \in \mathfrak{g}$.

\section{Ejemplo 1.}

1. La función identidad $i_{d}: X \rightarrow X$ es $\left(\mathfrak{g}, \mathfrak{g}^{\prime}\right)$ continua, si y sólo si, $\mathfrak{g}^{\prime} \subseteq \mathfrak{g}$. En efecto, $i_{d}$ es $\left(\mathfrak{g}, \mathfrak{g}^{\prime}\right)$-continua, si y sólo si, para cada $V \in \mathfrak{g}^{\prime}$ se tiene que $V=i_{d}^{-1}(V) \in \mathfrak{g}$.

2. Sea $\left\{\mathfrak{g}_{\alpha}\right\}_{\alpha \in \Lambda}$ una colección de $E D G$ 's sobre $X$. Si $f: X \rightarrow Y$ es una función $\left(\mathfrak{g}_{\alpha}, \mathfrak{g}^{\prime}\right)$-continua para todo $\alpha \in \Lambda$, entonces para todo $V \in \mathfrak{g}^{\prime}$ se tiene que $f^{-1}(V) \in \mathfrak{g}_{\alpha}$. Así $f^{-1}(V) \in \cap_{\alpha \in \Lambda} \mathfrak{g}_{\alpha}$ y por tanto $f$ es $\left(\mathfrak{g}, \mathfrak{g}^{\prime}\right)$-continua, donde $\mathfrak{g}=$ $\cap_{\alpha \in \Lambda} \mathfrak{g}_{\alpha}$.

La caracterización natural de continuidad en términos de subconjuntos $\mathfrak{g}$-cerrados también puede darse en este contexto, como se muestra en la siguiente proposición.

Proposición 3. Sean g y g g' $E D G$ 's sobre $X$ e $Y$ respectivamente. Una función $f: X \rightarrow Y$ es $\left(\mathfrak{g}, \mathfrak{g}^{\prime}\right)$-continua, si sólo si, la imagen recíproca de cualquier $\mathfrak{g}^{\prime}$-cerrado es un conjunto $\mathfrak{g}$-cerrado.

$\mathrm{Al}$ igual que en [6] y [8], puede definirse otra noción de continuidad entre $E D G^{\prime} s$. Esta noción fue orginalmente introducida en [5] en el año 1999.

Definición 4. Sean $\mathfrak{g}$ y $\mathfrak{g}^{\prime} E D G$ 's sobre $X$ e $Y$ respectivamente. Se dice que una función $f: X \rightarrow Y$ es $\mathcal{G}$-continua, si para cada $x \in X$ y cada $V \in \mathfrak{g}^{\prime}$ con $f(x) \in V$, existe $U \in \mathfrak{g}$ tal que $x \in U$ y $f(U) \subseteq V$.

En el caso topológico ó más generalmente en contextos donde la colección de abiertos es cerrada para uniones, estas dos definiciones de continuidad resultan ser equivalentes. Sin embargo, cuando las extendemos a conjuntos con $E D G^{\prime} s$ se tiene solamente una implicación como se muestra a continuación. La otra implicación se tiene asumiendo que la $E D G$ del dominio de la función es cerrada para uniones.
Proposición 4. Sean $\mathfrak{g}$ y $\mathfrak{g}^{\prime} E D G$ 's sobre $X$ e $Y$ respectivamente. Si la función $f: X \rightarrow Y$ es $\left(\mathfrak{g}, \mathfrak{g}^{\prime}\right)$-continua, entonces también es $\mathcal{G}$-continua. El recíproco es cierto si $\mathfrak{g}$ es cerrada para uniones arbitrarias.

Demostración. Supongamos que $f: X \rightarrow Y$ es $\left(\mathfrak{g}, \mathfrak{g}^{\prime}\right)$-continua y sean $x \in X$ y $V \in \mathfrak{g}^{\prime}$ tales que $f(x) \in V$. Entonces $f^{-1}(V) \in \mathfrak{g}$ y $x \in f^{-1}(V)$, donde $f\left(f^{-1}(V)\right) \subseteq V$. Por tanto, $f$ es $\mathcal{G}$-continua.

Recíprocamente, sean $x \in X$ y $V \in \mathfrak{g}^{\prime}$ tales que $x \in f^{-1}(V)$. Luego $f(x) \in V$. Como $f$ es $\mathcal{G}$-continua, existe $U \in \mathfrak{g}$ tal que $x \in U$ y $f(U) \subseteq V$ lo que implica que $x \in U \subseteq f^{-1}(V)$. Así $f^{-1}(V)$ es unión de $\mathfrak{g}$-abiertos y por la hipótesis, $f^{-1}(V) \in \mathfrak{g}$.

Siguiendo la demostración del resultado anterior se obtiene la siguiente caracterización de las funciones $\mathcal{G}$-continuas. Esta proposición muestra que la diferencia entre las dos nociones de continuidad descansa básicamente en si las colecciones consideradas son o no cerradas para uniones.

Proposición 5. Sean $\mathfrak{g}$ y $\mathfrak{g}^{\prime} E D G$ 's sobre $X$ e $Y$ respectivamente y $f: X \rightarrow Y$ una función. Las siguientes afirmaciones son equivalentes:

1. $f$ es $\mathcal{G}$-continua.

2. Para todo $\mathfrak{g}^{\prime}$-abierto $A$ se tiene que $f^{-1}(A)$ es unión de g-abiertos.

3. Para todo $\mathfrak{g}^{\prime}$-cerrado $C$ se tiene que $f^{-1}(C)$ es intersección de $\mathfrak{g}$-cerrados.

Ejemplo 2. En $\mathbb{R}$, la función $i_{d}: \mathbb{R} \rightarrow \mathbb{R}$ donde $\mathfrak{g}=\{\{x\}: x \in \mathbb{R}\}$ y $\mathfrak{g}^{\prime}=\{(a, b): a, b \in \mathbb{R}\}$, es una función $\mathcal{G}$-continua. Sin embargo, para $(a, b) \in \mathfrak{g}^{\prime}$ se tiene que $i_{d}^{-1}((a, b))=(a, b)$ no es un conjunto $\mathfrak{g}-$ abierto. Por consiguiente $i_{d}$ no es $\left(\mathfrak{g}, \mathfrak{g}^{\prime}\right)$-continua. Esto muestra que la $\mathcal{G}$-continuidad no implica, en general, la $\left(\mathfrak{g}, \mathfrak{g}^{\prime}\right)$-continuidad.

Recordemos que en topología la continuidad está caraterizada por los operadores interior y clausura. En nuestro caso, estas nociones caraterizan las funciones $\mathcal{G}$-continuas como se hace en [6] para los $m$-espacios y en [5] para colecciones.

Proposición 6. Sean g y g $\mathfrak{g}^{\prime} E D G$ 's sobre $X$ e $Y$ respectivamente y $f: X \rightarrow Y$ una función. Las siguientes afirmaciones son equivalentes: 
1. $f$ es $G$-continua.

2. $f^{-1}(V)=i_{\mathfrak{g}}\left(f^{-1}(V)\right)$ para todo $V \in \mathfrak{g}^{\prime}$.

3. $f\left(c_{\mathfrak{g}}(A)\right) \subseteq c_{\mathfrak{g}^{\prime}}(f(A))$ para todo $A \subseteq X$.

4. $c_{\mathfrak{g}}\left(f^{-1}(B)\right) \subseteq f^{-1}\left(c_{\mathfrak{g}^{\prime}}(B)\right)$ para todo $B \subseteq Y$.

5. $f^{-1}\left(i_{\mathfrak{g}^{\prime}}(B)\right) \subseteq i_{\mathfrak{g}}\left(f^{-1}(B)\right)$ para todo $B \subseteq Y$.

6. $f^{-1}(F)=c_{\mathfrak{g}}\left(f^{-1}(F)\right)$ para todo $F^{c} \in \mathfrak{g}^{\prime}$.

Demostración. $1 \Rightarrow 2$. Sea $V \in \mathfrak{g}^{\prime}$ y $x \in f^{-1}(V)$. Entonces $f(x) \in V$ y existe $U \in \mathfrak{g}$ tal que $x \in U$ y $f(U) \subseteq V$. Así $x \in U \subseteq f^{-1}(V)$. Esto implica que $f^{-1}(V) \subseteq i_{\mathfrak{g}}\left(f^{-1}(V)\right)$. Por la Proposición 1, tenemos que $i_{\mathfrak{g}}\left(f^{-1}(V)\right) \subseteq f^{-1}(V)$. Por lo tanto $f^{-1}(V)=i_{\mathfrak{g}}\left(f^{-1}(V)\right)$.

$2 \Rightarrow 3$. Sean $A \subseteq X, x \in c_{\mathfrak{g}}(A)$ y $V \in \mathfrak{g}^{\prime}$ tal $f(x) \in V$. Entonces $x \in f^{-1}(V)=i_{\mathfrak{g}}\left(f^{-1}(V)\right)$. Por la Proposición 2, existe $U \in \mathfrak{g}$ tal que $x \in U \subseteq f^{-1}(V)$ y $U \cap A \neq \emptyset$. Entonces $\emptyset \neq f(U \cap A) \subseteq f(U) \cap f(A) \subseteq V \cap f(A)$. Por consiguiente, $f(x) \in c_{\mathfrak{g}^{\prime}}(f(A))$ y por tanto $f\left(c_{\mathfrak{g}}(A)\right) \subseteq c_{\mathfrak{g}^{\prime}}(f(A))$.

$3 \Rightarrow 4$. Sea $B \subseteq Y$, entonces $f\left(c_{\mathrm{g}}\left(f^{-1}(B)\right)\right) \subseteq$ $c_{\mathfrak{g}^{\prime}}\left(f\left(f^{-1}(B)\right)\right) \subseteq c_{\mathfrak{g}^{\prime}}(B)$. Por lo tanto $c_{\mathfrak{g}}\left(f^{-1}(B)\right) \subseteq$ $f^{-1}\left(c_{\mathfrak{g}^{\prime}}(B)\right)$.

$4 \Rightarrow 5$. Sea $B \subseteq Y$, entonces $\left(i_{\mathfrak{g}}\left(f^{-1}(B)\right)\right)^{c}=$ $c_{\mathfrak{g}}\left(f^{-1}\left(B^{c}\right)\right) \subseteq f^{-1}\left(c_{\mathfrak{g}^{\prime}}\left(B^{c}\right)\right)=f^{-1}\left(\left(i_{\mathfrak{g}^{\prime}}(B)\right)^{c}\right)=$ $\left(f^{-1}\left(i_{\mathfrak{g}^{\prime}}(B)\right)\right)^{c}$. Por lo tanto $f^{-1}\left(i_{\mathfrak{g}^{\prime}}(B)\right) \subseteq i_{\mathfrak{g}}\left(f^{-1}(B)\right)$.

\begin{tabular}{cccccc}
$5 \Rightarrow 6$. & Sea $K \subseteq$ & \multicolumn{1}{c}{ tal } & que \\
$K^{c} \in \quad \mathfrak{g}^{\prime}$. & Entonces & $\left(f^{-1}(K)\right)^{c}$ & $=$ \\
$f^{-1}\left(i_{\mathfrak{g}^{\prime}}\left(K^{c}\right)\right) \subseteq$ & $i_{\mathfrak{g}}\left(f^{-1}\left(K^{c}\right)\right)=i_{\mathfrak{g}}\left(\left(f^{-1}(K)\right)^{c}\right)$ & $=$ \\
$\left(c_{\mathfrak{g}}\left(f^{-1}(K)\right)\right)^{c}$. & Por lo tanto $c_{\mathfrak{g}}\left(f^{-1}(K)\right)$ & $\subseteq$ \\
$f^{-1}(K) \subseteq c_{\mathfrak{g}}\left(f^{-1}(K)\right)$. Así $c_{\mathfrak{g}}\left(f^{-1}(K)\right)=f^{-1}(K)$.
\end{tabular}

$6 \Rightarrow 1$. Sean $x \in X$ y $V \in \mathfrak{g}^{\prime}$ tales que $f(x) \in V$. Por 6. tenemos que $\left(f^{-1}(V)\right)^{c}=f^{-1}\left(V^{c}\right)=c_{\mathfrak{g}}\left(f^{-1}\left(V^{c}\right)\right)=$ $c_{\mathfrak{g}}\left(\left(f^{-1}(V)\right)^{c}\right)=\left(i_{\mathfrak{g}}\left(f^{-1}(V)\right)\right)^{c}$. Así $f^{-1}(V)=$ $i_{\mathfrak{g}}\left(f^{-1}(V)\right)$. En consecuencia, existe $U \in \mathfrak{g}$ tal que $x \in U \subseteq f^{-1}(V)$. Por lo tanto $x \in U$ y $f(U) \subseteq V$.

El siguiente resultado es familiar y nos indica que los dos tipos de continuidad definidas en conjuntos con $E D G^{\prime} s$ se preservan bajo composición de funciones.
Proposición 7. Sean $\mathfrak{g}$, $\mathfrak{g}^{\prime}$ y $\mathfrak{g}^{\prime \prime} E D G$ 's sobre $X$, $Y$ y $Z$ respectivamente. Si $f: X \rightarrow Y$ es una función $\left(\mathfrak{g}, \mathfrak{g}^{\prime}\right)$-continua $(\mathcal{G}$-continua) y $h: Y \rightarrow Z$ es una función $\left(\mathfrak{g}^{\prime}, \mathfrak{g}^{\prime \prime}\right)$-continua $(\mathcal{G}$-continua), entonces la función $h \circ f: X \rightarrow Z$ es $\left(\mathfrak{g}, \mathfrak{g}^{\prime \prime}\right)$-continua (G-continua).

Demostración. Si $V \in \mathfrak{g}^{\prime \prime}$, entonces $h^{-1}(V) \in \mathfrak{g}^{\prime}$. Así $f^{-1}\left(h^{-1}(V)\right) \in \mathfrak{g}$. Ahora bien, $(h \circ f)^{-1}(V)=$ $f^{-1}\left(h^{-1}(V)\right) \in \mathfrak{g}$. En consecuencia, $h \circ f$ es $\left(\mathfrak{g}, \mathfrak{g}^{\prime \prime}\right)$-continua.

Por otro lado, sean $x \in X$ y $U \in \mathfrak{g}^{\prime \prime}$ tales que ( $h \circ$ $f)(x)=h(f(x)) \in U$. Entonces, existe $V \in \mathfrak{g}^{\prime}$ tal que $f(x) \in V$ y $h(V) \subseteq U$. Además, existe $W \in \mathfrak{g}$ tal que $x \in W$ y $f(W) \subseteq V$. Por lo tanto, $x \in W$ y $(h \circ f)(W) \subseteq$ $U$.

\section{Otras propiedades}

En esta sección se presentan algunos resultados que relacionan la continuidad y algunos axiomas de separación. Análogamente a la definición de espacios $T_{0}, T_{1}$ y $T_{2}$ usando abiertos, se tienen los respectivos axiomas $\mathfrak{g}-T_{0}, \mathfrak{g}-T_{1}, \mathfrak{g}-T_{2}$ usando $\mathfrak{g}$-abiertos. Estos resultados extienden resultados bien conocidos en topología, en $m$-espacios [6] y en topologías generalizadas [8].

Teniendo en cuenta que un espacio es $\mathfrak{g}-T_{1}$ si y solo si cada conjunto unitario es intersección de conjuntos $\mathfrak{g}$-cerrados, obtenemos otra caracterización para los espacios $\mathfrak{g}-T_{1}$.

Proposición 8. Un espacio $Y$ es $\mathfrak{g}-T_{1}$ si y solo si para cada función $\mathcal{G}$-continua $\left(\left(\mathfrak{g}, \mathfrak{g}^{\prime}\right)\right.$-continua) $f: X \rightarrow Y, f^{-1}(\{y\})$ es intersección de $\mathfrak{g}$-cerrados para cada $y \in Y$.

Demostración. La suficiencia es clara ya que si $y \in$ $Y$, entonces $\{y\}$ es intersección de $\mathfrak{g}$-cerrados y por tanto $f^{-1}(\{y\})$ es intersección de $\mathfrak{g}$-cerrados por la $G$-continuidad de $f$. Para el recíproco basta tomar la función identidad $i_{Y}: Y \rightarrow Y$. Con esto se tiene que para cada $y \in Y, i_{Y}^{-1}(\{y\})=\{y\}$ es intersección de g-cerrados. Así, $Y$ es $\mathfrak{g}-T_{1}$.

Proposición 9. Sean g y $\mathfrak{g}^{\prime} E D G$ 's sobre $X$ e $Y$ respectivamente con $Y$ un espacio $\mathfrak{g}^{\prime}-T_{1}$ y $f: X \rightarrow Y$ una función $G$-continua $\left(\left(\mathfrak{g}, \mathfrak{g}^{\prime}\right)\right.$-continua). Si para $A \subseteq X, f(A)=\{y\}$ para algún $y \in Y$, entonces $f\left(c_{\mathfrak{g}}(A)\right)=\{y\}$. 
Demostración. Basta observar que si $f(A)=\{y\}$, entonces $A \subseteq f^{-1}(\{y\})$. Como $Y$ es $\mathfrak{g}^{\prime}-T_{1}$ entonces $\{y\}$ es intersección de $\mathfrak{g}^{\prime}$-cerrados. Así $f^{-1}(\{y\})$ es intersección de $\mathfrak{g}$-cerrados (Proposición 5). Luego $c_{\mathfrak{g}}(A) \subseteq f^{-1}(\{y\})$ y esto implica que $f\left(c_{\mathfrak{g}}(A)\right) \subseteq\{y\}$. Es decir, $f\left(c_{\mathfrak{g}}(A)\right)=\{y\}$ ya que $f(A) \subseteq f\left(c_{\mathfrak{g}}(A)\right)$.

Proposición 10. Sean g y g $\mathfrak{g}^{\prime} E D G$ 's sobre $X$ e $Y$ respectivamente y $f: X \rightarrow Y$ una función $G$-continua $\left(\left(\mathfrak{g}, \mathfrak{g}^{\prime}\right)-\right.$ continua $)$. Si $Y$ es $\mathfrak{g}^{\prime}-T_{2}\left(\mathfrak{g}^{\prime}-T_{0}, \mathfrak{g}^{\prime}-T_{1}\right)$ y $f$ es inyectiva, entonces $X$ es $\mathfrak{g}-T_{2}\left(\mathfrak{g}-T_{0}, \mathfrak{g}-T_{1}\right)$.

Demostración. Sean $x, y$ dos puntos distintos de $X$. Entonces $f(x) \neq f(y)$. Si $Y$ es $\mathfrak{g}^{\prime}-T_{2}$, existen $\mathfrak{g}^{\prime}$-abiertos disjuntos $U$ y $V$ tales que $f(x) \in U$ y $f(y) \in V$. Por hipótesis existen $\mathfrak{g}$-abiertos $U_{x} \mathrm{y} V_{y}$ tales que $x \in U_{x}, y \in U_{y}, f\left(U_{x}\right) \subseteq U$ y $f\left(V_{y}\right) \subseteq V$. Como $f\left(U_{x}\right) \cap f\left(V_{y}\right) \subseteq U \cap V=\emptyset$, entonces se concluye que $X$ es $\mathfrak{g}-T_{2}$. La otra parte de la prueba se hace análogamente.

El resto de la demostración es consecuencia directa del hecho que toda función $\left(\mathfrak{g}, \mathfrak{g}^{\prime}\right)$-continua es G-continua (Proposición 4).

Dadas las EDG's $\mathfrak{g}$ y $\mathfrak{g}^{\prime}$ sobre $X$ e $Y$ respectivamente y $f: X \rightarrow Y$, diremos que $f$ es $\mathfrak{g}$-abierta si la imagen de cualquier $\mathfrak{g}$-abierto en $X$ es unión de $\mathfrak{g}^{\prime}$-abiertos en $Y$. Con esto puede probarse fácilmente el siguiente resultado.

Proposición 11. Sean g y $\mathfrak{g}^{\prime} E D G$ 's sobre $X$ e $Y$ respectivamente y $f: X \rightarrow Y$ una función biyectiva y $\mathfrak{g}$-abierta. Si $X$ es $\mathfrak{g}-T_{2}\left(\mathfrak{g}-T_{0}, \mathfrak{g}-T_{1}\right)$, entonces $Y$ es $\mathfrak{g}^{\prime}-T_{2}\left(\mathfrak{g}^{\prime}-T_{0}, \mathfrak{g}^{\prime}-T_{1}\right)$.

Recordemos que dada una función $f: X \rightarrow Y$ el grafo de $f$ está definido como el conjunto $G(f)=$ $\{(x, f(x)): x \in X\}$ y el kernel de $f$ está definido como $\operatorname{Ker}(f)=\left\{\left(x, x^{\prime}\right): f(x)=f\left(x^{\prime}\right)\right\}$. Estos dos conjuntos juegan un papel muy importante en algunos resultados topológicos relacionados con los espacios $T_{2}$.

Si $\mathfrak{g}$ y $\mathfrak{g}^{\prime}$ son $E D G$ 's sobre $X$ e $Y$ respectivamente, entonces sobre $X \times Y$ consideramos la $E D G$ dada por el conjunto $\mathfrak{g} \times \mathfrak{g}^{\prime}=\left\{A \times B: A \in \mathfrak{g}\right.$ y $\left.B \in \mathfrak{g}^{\prime}\right\}$.

Proposición 12. Sean g y $\mathfrak{g}^{\prime} E D G$ 's sobre $X$ e $Y$ respectivamente y $f: X \rightarrow Y$ una función $\mathcal{G}$-continua $\left(\left(\mathfrak{g}, \mathfrak{g}^{\prime}\right)-\right.$ continua). Si $Y$ es $\mathfrak{g}^{\prime}-T_{2}$, entonces $G(f)$ es intersección de $(\mathfrak{g} \times$ $\left.\mathfrak{g}^{\prime}\right)$-cerrados en $X \times Y$ y $\operatorname{Ker}(f)$ es intersección de $(\mathfrak{g} \times \mathfrak{g})$-cerrados en $X \times X$.

Demostración. Sea $(x, y) \in G(f)^{c}$. Entonces $y \neq f(x)$ y como $Y$ es $\mathfrak{g}^{\prime}-T_{2}$, existen $\mathfrak{g}^{\prime}$-abiertos disjuntos $U$ y $V$ tales que $y \in U$ y $f(x) \in V$. Como $f$ es $G$-continua existe $V_{x} \in \mathfrak{g}$ tal que $x \in V_{x}$ y $f\left(V_{x}\right) \subseteq V$. Como $G(f) \cap\left(V_{x} \times U\right)=\emptyset$ entonces $V_{x} \times U \subseteq G(f)^{c}$. Así todo punto de $G(f)^{c}$ es $\left(\mathfrak{g} \times \mathfrak{g}^{\prime}\right)$-interior y esto implica que $G(f)^{c}$ es unión de $\left(\mathfrak{g} \times \mathfrak{g}^{\prime}\right)$-abiertos ó equivalentemente $G(f)$ es intersección de $\left(\mathfrak{g} \times \mathfrak{g}^{\prime}\right)$ cerrados.

Si $(x, y) \in \operatorname{Ker}(f)^{c}$ entonces $f(x) \neq f(y)$. Luego existen $\mathfrak{g}^{\prime}$-abiertos disjuntos $U, V$ tales que $f(x) \in$ $U$ y $f(y) \in V$. Como $f$ es $\mathcal{G}$-continua existen $\mathfrak{g}$-abiertos $U_{x}$ y $V_{y}$ tales que $x \in U_{x}, f\left(U_{x}\right) \subseteq U$, $y \in V_{y}$ y $f\left(V_{y}\right) \subseteq V$. Además puede verse fácilmente que $\operatorname{Ker}(f) \cap\left(U_{x} \times V_{y}\right)=\emptyset$. Así $U_{x} \times V_{y} \subseteq \operatorname{Ker}(f)^{c}$ y esto implica que $\operatorname{Ker}(f)$ es intersección de $(\mathfrak{g} \times \mathfrak{g})$ cerrados.

Proposición 13. Sean $\mathfrak{g}$ y $\mathfrak{g}^{\prime} E D G$ 's sobre $X$ e $Y$ respectivamente y $f: X \rightarrow Y$ una función sobreyectiva y g-abierta. Si $\operatorname{Ker}(f)$ es intersección de $(\mathfrak{g} \times \mathfrak{g})$ cerrados, entonces $Y$ es $\mathfrak{g}^{\prime}-T_{2}$.

Demostración. Sean $y_{1}, y_{2} \in Y$ con $y_{1} \neq y_{2}$. Entonces existen $x_{1}, x_{2} \in X$ tales que $f\left(x_{1}\right)=y_{1}$ y $f\left(x_{2}\right)=y_{2}$. Así $\left(x_{1}, x_{2}\right) \in \operatorname{Ker}(f)^{c}$ y es claro que $\operatorname{Ker}(f)$ es unión de conjuntos $(\mathfrak{g} \times \mathfrak{g})$-abiertos. Entonces existen $\mathfrak{g}$ abiertos $A$ y $B$ tales que $x_{1} \in A$ y $x_{2} \in B$. Esto implica que $y_{1} \in f(A)$ y $y_{2} \in f(B)$ y cada conjunto $f(A)$ y $f(B)$ es unión de $\mathfrak{g}^{\prime}-$ abiertos. Entonces existen $\mathfrak{g}^{\prime}$ abiertos $U, V$ con $U \subseteq f(A)$ y $V \subseteq f(B)$ y tales que $y_{1} \in U$ y $y_{2} \in V$. Como $f(A) \cap f(B)=\emptyset$, entonces $U \cap V=\emptyset$ y así $Y$ es $\mathfrak{g}^{\prime}-T_{2}$.

Dadas las EDG's $\mathfrak{g}$ y $\mathfrak{g}^{\prime}$ sobre $X$ e $Y$ respectivamente y las funciones $f, g: X \rightarrow Y$, el igualador de $f y g$ es el conjunto $e q(f, g)=\{x \in X: f(x)=g(x)\}$.

Proposición 14. Sean g y g $\mathfrak{g}^{\prime} E D G$ 's sobre $X$ e $Y$ respectivamente y $f, g: X \rightarrow Y$ funciones $G$-continuas $\left(\left(\mathfrak{g}, \mathfrak{g}^{\prime}\right)-\right.$ continuas $)$. Si $Y$ es $\mathfrak{g}^{\prime}-T_{2}$, entonces el conjunto $e q(f, g)$ es intersección de $\mathfrak{g}$-cerrados.

Demostración. Si $z \in e q(f, g)^{c}$ entonces $f(z) \neq g(z)$ y por tanto existen $\mathfrak{g}^{\prime}$-abiertos disjuntos $U, V$ tales que $f(z) \in U$ y $g(z) \in V$. Usando el hecho que $f$ y $g$ son $\mathcal{G}$-continuas encontramos g-abiertos $U_{z}$ y $V_{z}$ 
tales que $z \in U_{z} \cap V_{z} \subseteq e q(f, g)^{c}$. Es decir, el conjunto $e q(f, g)^{c}$ es unión de g-abiertos, lo cual implica que $e q(f, g)$ es intersección de g-cerrados.

Si $X$ es un g-espacio, entonces $A \subseteq X$ se llama $\mathfrak{g}$-denso si $c_{\mathfrak{g}}(A)=X$. Bajo las hipótesis de la proposición anterior si $f(a)=g(a)$ para todo $a \in A$ entonces $A \subseteq e q(f, g)$. Si $e q(f, g)^{c} \neq \emptyset$ entonces existe un g-abierto $V \neq \emptyset$ tal que $A \cap V=\emptyset$. Así $A$ no sería g-denso. Por tanto $e q(f, g)=X$ y así $f=g$. Es decir, $f$ y $g$ están completamente determinadas por sus valores en conjuntos $\mathfrak{g}$-densos.

Un caso particular de la proposición anterior consiste en considerar $X$ un espacio $\mathfrak{g}-T_{2}$ y $f: X \rightarrow X$ una función $\mathcal{G}$-continua, entonces se tiene que el conjunto $\{x \in X: f(x)=x\}$ es intersección de $\mathfrak{g}$-cerrados. Es decir, el conjunto de puntos fijos de una función $\mathcal{G}$-continua de un espacio $\mathfrak{g}-T_{2}$ en sí mismo, es intersección de $\mathfrak{g}$-cerrados.

\section{Referencias}

[1] A. Császár, "Generalized open sets", Acta Math. Hungar., vol. 75, no. 1-2, pp. 65-87, 1997.

[2] H. Maki, On generalizing semi-open and preopen sets, Report for Meeting on Topolo- gical Spaces and its Applications, Yatsushiro College of Technology, 1996.

[3] A. Császár, "Weak structures", Acta Math. Hungar, vol. 131, no. 1-2, pp. 193-195, 2011.

[4] J. Ávila and F. Molina, "Generalized weak structures", Int. Math. Forum, vol. 7, no. 52, pp. 2589-2595, 2012.

[5] A. Donado, Topología y colecciones, Universidad Pedagógica Nacional, Bogotá, 1999.

[6] V. Popa and T. Noiri, "On M-continuous functions", Anal. Univ. Dunarea de Jos Galati, Ser. Mat Fiz. Mec. Teor. , Fasc II, vol. 18, no. 23, pp. 31-41, 2000.

[7] A. Császár, "Generalized topology, generalized continuity", Acta Math. Hungar., vol. 96, no. 4, pp. 351-357, 2002.

[8] W. Min, "Weak continuity on generalized topological spaces", Acta. Math. Hungar., vol. 124, no. 1-2, pp. 73-81, 2009.

[9] W. Min, "Generalized continuous functions defined by generalized open sets on generalized topological spaces", Acta. Math. Hungar, vol. 128, no. 4, pp. 299-306, 2010. 